\title{
Espirometría incentiva preoperatoria para mejorar el tiempo de tolerancia a la desaturación durante la inducción anestésica en pacientes con obesidad
}

\section{Preoperative incentive spirometry to improve desaturation tolerance time during anesthetic induction in obese patients}

\author{
Susy Saez Pavía Vega, ${ }^{\star}$ Nora Bernal Ríos, ${ }^{\ddagger}$ Armando Torres Gómez, ${ }^{\S}$ \\ María Fernanda Alarcón Trejo, ${ }^{*}$ Lizbeth Falcón Valdez*
}

Citar como: Pavía VSS, Bernal RN, Torres GA, Alarcón TMF, Falcón VL. Espirometría incentiva preoperatoria para mejorar el tiempo de tolerancia a la desaturación durante la inducción anestésica en pacientes con obesidad. An Med ABC. 2021; 66 (3): 169-176. https:// dx.doi.org/10.35366/101663

\section{RESUMEN}

Introducción: Los pacientes con obesidad tienen una capacidad funcional residual disminuida y su tiempo de tolerancia a la desaturación es menor. La espirometría incentiva imita de forma natural los suspiros y bostezos, llevando al paciente a realizar respiraciones largas, lentas y profundas, disminuyendo la presión pleural, mejorando la expansión torácica y el intercambio gaseoso. Objetivo: Evaluar la eficacia de la espirometría incentiva preoperatoria en comparación con control para mejorar el tiempo de tolerancia a la desaturación hasta $90 \%$ en pacientes con obesidad sometidos a cirugía electiva. Material y métodos: Estudio prospectivo, aleatorizado, simple, abierto, en el que se estudiaron 24 pacientes, 12 con espirometría incentiva preoperatoria y 12 para grupo control sin espirometría incentiva preoperatoria. Resultados: Desaturación a 90\% en el grupo 1 con media de 148.83 segundos y el grupo control con 113.08 segundos, diferencia de 35.75 segundos. Los datos obtenidos para recuperar una saturación a $94 \%$ muestran en un inicio ser favorables para los pacientes que realizaron espirometría, ya que la mediana es de 41 segundos, mientras que en el

\section{ABSTRACT}

Introduction: Patients with obesity have a diminished residual functional capacity and their time of tolerance to desaturation is lower. Incentive spirometry, naturally mimics sighs and yawns, leading the patient to perform long, slow, and deep breaths, decreasing pleural pressure, improving thoracic expansion and gas exchange. Objective: Evaluate the efficacy of preoperative incentive spirometry compared to control, to improve the time to tolerance to desaturation up to $90 \%$ in obese patients undergoing elective surgery. Material and methods: A simple open randomized prospective study, where 24 patients were studied, of which 12 patients with preoperative incentive spirometry and 12 patients for the control group without preoperative incentive spirometry. Results: Desaturation at 90\% in group 1 with mean of 148.83 seconds and control group with 113.08 seconds, difference of 35.75 seconds. The data obtained to recover a saturation to $94 \%$ show at first to be favorable for patients who performed spirometry, since the median is 41 seconds, while in the control group is 55. Conclusions: Obese patients under anesthesia general with orotracheal intubation would benefit from the use of preoperative Triflo routinely.
* Médico Residente de Anestesiología.

₹ Médico adscrito al Servicio de Anestesia y Terapia Intensiva.

$\S$ Cirujano Traumatólogo y Ortopedista, Maestro en Ciencias, Miembro del Sistema Nacional de Investigadores.
Correspondencia:

Dra. María Fernanda Alarcón Trejo

E-mail: feralarcontre@gmail.com 
grupo control es de 55. Conclusiones: Los pacientes obesos sometidos a anestesia general con intubación orotraqueal se verían beneficiados con el uso de Triflo preoperatorio de manera rutinaria.

Palabras clave: Espirometría incentiva, tiempo en desaturar, capacidad residual funcional.

Nivel de evidencia: II

Abreviaturas:

IMC = Índice de masa corporal.

$\mathrm{CRF}=$ Capacidad residual funcional.

CPAP = Presión positiva continua en la vía aérea.

$\mathrm{CAE}-\mathrm{HS}=$ Conducto auditivo externo-hueco supraesternal.

$\mathrm{ASA}=$ American Society of Anesthesiologists.

$\mathrm{FC}=$ Frecuencia cardiaca.

PAM $=$ Presión arterial media.

PEEP = Presión positiva al final de la espiración

$\mathrm{PANI}=$ Presión arterial no invasiva.

CAM = Concentración alveolar mínima .

$\mathrm{BIS}=$ Índice biespectral.

$E C G=$ Electrocardiograma.

\section{INTRODUCCIÓN}

De acuerdo con la Norma Oficial Mexicana para el tratamiento integral del sobrepeso y la obesidad (NOM-008-SSA3-2010), se define como la enfermedad caracterizada por el exceso de tejido adiposo en el organismo, la cual se determina cuando en las personas adultas existe un índice de masa corporal (IMC) igual o mayor a $30 \mathrm{~kg} / \mathrm{m}^{2}$ y en las personas adultas de estatura baja (menor de 1.50 metros en mujeres y de 1.60 metros en hombres) igual o mayor a $25 \mathrm{~kg} / \mathrm{m}^{2} .{ }^{1}$ Según las estadísticas del Sector Salud en México, se calcula que 12.1 millones de personas padecen obesidad, lo que afecta a $70 \%$ de la población mexicana entre los 30 y 60 años de edad (mujeres: $71.9 \%$ y hombres: $66.7 \%)^{1}$

\section{Fisiología respiratoria en la obesidad}

En los pacientes con obesidad existe una serie de alteraciones respiratorias que afectan a los volúmenes pulmonares y dentro de las más importantes se encuentran la distensibilidad y la relación ventilación/ perfusión, que ocasionan a su vez hipoxemia permanente por la ineficacia del trabajo respiratorio. ${ }^{2}$ Hay una disminución del volumen de reserva espiratorio proporcional al sobrepeso, con mantenimiento o incluso aumento del volumen residual, lo que origina una disminución de la capacidad funcional residual y aumento del riesgo de formación de atelectasias. ${ }^{3}$ Por otra parte, la distensibilidad torácica está redu-
Keywords: Incentive spirometry, time to desaturate, functional residual capacity.

Level of evidence: II

cida, con el consecuente aumento del trabajo respiratorio y la limitación del individuo para responder al aumento de la demanda ventilatoria. ${ }^{4}$ Las causas son el acúmulo de grasa a nivel costal, infradiafragmático e intraabdominal. ${ }^{5}$ La distensibilidad pulmonar se mantiene normal, excepto cuando la obesidad se hace de larga evolución, disminuyendo entonces, debido en parte al aumento de sangre en el parénquima pulmonar y en parte a la propia caída de la capacidad funcional residual (CFR). ${ }^{6}$ Además, se producen alteraciones del intercambio gaseoso por dos razones: hay zonas de espacio muerto (aumento de la relación ventilación/perfusión), originadas por las anomalías circulatorias y la vasoconstricción pulmonar hipoxémica; y zonas con efecto shunt (disminución de la relación ventilación/perfusión), debido al colapso alveolar producido por la disminución de la CFR y al aumento del volumen sanguíneo pulmonar.

Las alteraciones respiratorias se agravan con el cambio de postura de sedestación a decúbito supino. El desplazamiento cefálico del diafragma hace que la CFR, que en el obeso está muy próxima a la capacidad de cierre, disminuya, por lo que parte del ciclo respiratorio se realiza en el volumen de cierre, favoreciendo la aparición de atelectasias. ${ }^{7} \mathrm{El}$ riesgo de hipoxemia viene determinado por la disminución de la diferencia alvéolo-arterial de oxígeno, que, a su vez, es directamente proporcional a la disminución de la diferencia capacidad funcional residual-capacidad de cierre.

\section{Preoxigenación en el paciente obeso}

Es importante prevenir la desaturación arterial durante la apnea en la fase de intubación (el tiempo promedio en el que desciende la $\mathrm{SaO}_{2}$ a $90 \%$ en pacientes con peso corporal normal fue de seis minutos, en pacientes con obesidad grado I y II fue de 4.11 minutos, y en pacientes con obesidad mórbida tenían sólo 2.7 minutos), ${ }^{8}$ debido a las escasas reservas de oxígeno condicionadas por la CFR disminuida, ade- 
más de depender de otros factores como los niveles de hemoglobina o el metabolismo basal. La CFR se reduce aún más en posición supina y después de la inducción, llegando a ser de 1,900 mL comparado con los 2,600 mL del paciente no obeso. ${ }^{9}$ Con base en lo anterior, se acostumbra realizar técnicas convencionales para desnitrogenizar los pulmones.

También ha demostrado su utilidad la administración de presión con $10 \mathrm{cmH}_{2} \mathrm{O}$ de presión positiva continua en la vía aérea (CPAP) por cinco minutos antes de la inducción, seguida de $10 \mathrm{cmH}_{2} \mathrm{O}$ de CPAP por mascarilla facial antes de la intubación, lo que agrega un minuto antes de que ocurra la desaturación del paciente. ${ }^{10}$

Existe también la posición en rampa, la cual tiene como objetivo alinear el eje conducto auditivo externo-hueco supraesternal (CAE-HS) en el plano horizontal. Esta posición además de favorecer una mejor laringoscopia y condiciones de intubación permite una mejor ventilación, preoxigenación, oxigenación y tolerancia a la apnea. ${ }^{11}$

\section{Espirometría incentiva preoperatoria}

El incentivo respiratorio o Triflo como dis0positivo ha sido ampliamente utilizado en la práctica clínica, especialmente en la intervención de pacientes en el periodo pre- y postoperatorio de cirugías importantes, debido a su bajo costo, facilidad de aplicación y buena adherencia de los pacientes. ${ }^{12}$

Las bases fisiológicas de la espirometría incentiva reportadas por Agostini y Singh, se sustentan en el manejo de las atelectasias a través de la eliminación de las secreciones retenidas en la vía aérea y la reexpansión del parénquima pulmonar. ${ }^{13}$ La espirometría incentiva se asemeja a una inspiración máxima sostenida que se lleva a cabo a través de un dispositivo que proporciona una retroalimentación visual cuando el paciente inhala a cierta cantidad de flujo o volumen y lo sostiene por lo menos cinco segundos.

Objetivo: evaluar la eficacia de la espirometría incentiva preoperatoria en comparación con control para mejorar el tiempo de tolerancia a la desaturación hasta $90 \%$ en pacientes con obesidad sometidos a cirugía electiva.

\section{MATERIAL Y MÉTODOS}

La presente investigación cumple los lineamientos mencionados en la declaración de Helsinki y la Ley General de Salud.
El trabajo de investigación fue sometido a los Comités de Investigación y Ética en el Centro Médico $\mathrm{ABC}$, quedando debidamente registrado con la clave TABC-19-19.

Estudio prospectivo, aleatorizado, simple, abierto, que se realizó en el Centro Médico ABC. El tamaño de la muestra fue de 24 pacientes, 12 para el grupo control sin espirometría incentiva preoperatoria y 12 para espirometría incentiva preoperatoria. El tamaño de muestra se calculó a partir de la información presentada por Jense HG y colaboradores, ${ }^{8}$ quienes reportaron $364 \pm 24$ segundos de tolerancia a la desaturación en pacientes con IMC menor de 30 y $247 \pm$ 21 segundos en pacientes con obesidad grado I y II. Dixon BJ y colegas ${ }^{14}$ reportan que en pacientes con obesidad mórbida elevar la cabeza $25^{\circ}$, aumenta la tolerancia a la desaturación en 50 segundos. Después de hacer una búsqueda extensiva de la literatura, no encontramos el efecto de dicha maniobra en pacientes con obesidad I y II.

En este centro médico se aplica la posición de HELP o de rampa, que incluye la elevación de la cabeza de 20 a $30^{\circ}$ aproximadamente, para alinear el conducto auditivo externo con el hueco supraesternal. Asumimos que los pacientes con obesidad grado I y II a quienes se colocan en posición de HELP, se verán beneficiados y su tolerancia a la desaturación mejorará en al menos 50 segundos. Partiendo de estas asunciones, para calcular el tamaño de muestra, consideramos la media de tolerancia a la desaturación en pacientes normales $(=364 \pm 24)$ y para pacientes con obesidad I y II $(=297 \pm 21)$ (sumando los 50 segundos por la maniobra de HELP). La diferencia entre estos valores da un tamaño del efecto de 2.97 (de Cohen). Considerando una prueba t para grupos independientes a dos colas, con un nivel de significancia de 0.05 , con un poder de $99 \%$ y asignación simétrica a los dos grupos, obtuvimos un tamaño de muestra de seis pacientes por grupo. Para aumentar la robustez del estudio, decidimos duplicar el tamaño de muestra quedando de 12 pacientes por grupo, es decir, 24 pacientes en total.

\section{Criterios de inclusión}

Pacientes sometidos a cirugía electiva durante el periodo comprendido del 1 de marzo de 2018 al 30 de junio de 2018, en el Centro Médico ABC, edad 18 a 75 años, ASA II, con estabilidad hemodinámica y respiratoria, con ayuno de ocho horas, IMC 30-39. Además, es requisito que el Comité de Investigación y Ética apruebe el protocolo. 


\section{Criterios de exclusión}

1. Portadores de cardiopatías o neumopatías no controladas.

2. Cirugías de urgencia.

3. Inestabilidad hemodinámica.

\section{Metodología}

El estudio se dividió en dos grupos: el grupo experimental con uso de espirometría incentiva preoperatoria y el grupo control sin espirometría incentiva preoperatoria. Los sujetos fueron asignados a los grupos de estudio por medio de un procedimiento de aleatorización simple con reemplazo.

Se trató de pacientes programados, sometidos a anestesia general con intubación orotraqueal. Los pacientes del grupo 1 fueron asignados para realizar espirometría incentiva preoperatoria con un mínimo de una hora antes de la inducción anestésica, el ejercicio respiratorio fue elaborado con la ayuda de un Triflo brindado por enfermería, su modo de uso fue explicado en todos los casos por la médico residente de anestesiología. Los pasos a seguir eran cumplir inspiraciones máximas sostenidas, llevando a cabo una retroalimentación cuando el paciente inhalaba un predeterminado flujo y sostenía la inflación durante al menos cinco segundos. Se instruyó al paciente para mantener el espirómetro en una posición vertical, exhalar normalmente y luego colocar los labios alrededor de la boquilla. El siguiente paso era la inhalación para elevar la pelota en la cámara a la meta fijada, de preferencia las tres pelotas. A inhalación máxima, se retiraba la boquilla, seguido por una apnea y la espiración normal. La duración de la terapia debía ser de 30 minutos en total.

Una vez que los pacientes se encontraban en el quirófano, la monitorización fue con ECG de cinco derivaciones, PANI, pletismografía, capnografía, espirometría, analizador de gases, CAM, BIS o entropía y clínica. Se registraron FC y PAM iniciales. Se inició en ambos grupos preoxigenación colocando al paciente en posición de HELP (alineando el conducto auditivo externo con el hueco supraesternal), mascarilla facial con ajuste hermético, $\mathrm{FiO}_{2}$ a $80 \%$, flujo total 6 $\mathrm{L} /$ min y CPAP $10 \mathrm{cmH}_{2} \mathrm{O}$ durante tres minutos para lograr un valor de $\mathrm{EtO}_{2}$ excedente o igual a $90 \%$.

Se realizó una inducción anestésica para secuencia rápida con propofol (1-3 mg/kg), fentanil (1-5 $\mu \mathrm{g} /$ $\mathrm{kg}$ ), rocuronio dosis a 4ED95 calculado por peso magro $(1-1.2 \mathrm{mg} / \mathrm{kg}$ ) y lidocaína $(0.5-1 \mathrm{mg} / \mathrm{kg})$. Al minuto de latencia del relajante muscular, se retiraba la mascarilla facial y se iniciaba cuenta cronometrada del tiempo de tolerancia a la desaturación, al mismo tiempo el operador principal realizaba la intubación orotraqueal con videolaringoscopio para descartar colocación esofágica de la cánula, ya que el dispositivo no se conectaría al circuito de anestesia hasta llegar a la saturación deseada de $90 \%$. Mientras el paciente se encontraba en apnea se registraron nue-

Tabla 1: Características de la población.

\begin{tabular}{|c|c|c|c|}
\hline Característica & Grupo 1: Triflo ( $\mathrm{N}=12)$ & Grupo 2: control $(\mathrm{N}=12)$ & $p^{*}$ \\
\hline Sexo, $n(\%)$ & & & 1.0000 \\
\hline Masculino & $7(58.33)$ & $7(58.33)$ & \\
\hline $\mathrm{IMC}$, media $\pm \mathrm{DE}$ & $32.20 \pm 1.42$ & $32.71 \pm 2.83$ & 0.5836 \\
\hline Circunferencia de cuello, mediana (RIC, mín.-máx.) & $44.5(2,37-46)$ & $44.5(4.25,34-48)$ & 0.5598 \\
\hline ASA II, $n(\%)$ & $12(100.00)$ & $12(100.00)$ & 1.000 \\
\hline Tipo de cirugía, $n(\%)$ & & & 0.4076 \\
\hline Cirugía general & $6(50.00)$ & $8(66.67)$ & \\
\hline Ortopedia & $6(50.00)$ & $4(33.33)$ & \\
\hline Laparoscopia & $2(16.67)$ & $4(33.33)$ & $0.0239^{* *}$ \\
\hline PEEP, media \pm DE & $6.33 \pm 0.98$ & $6.67 \pm 0.89$ & 0.3932 \\
\hline
\end{tabular}

IMC = índice de masa corporal; RIC = rango intercuartil; $A S A$ = sistema de clasificación que utiliza la American Society of Anesthesiologists para estimar el riesgo que plantea la anestesia para los distintos estados del paciente; PEEP = presión positiva al final de la espiración; EtCO ${ }_{2}=$ concentración máxima de dióxido de carbono espirado durante un ciclo respiratorio.

* Prueba t de Student, $\chi^{2}$, suma de rangos de Wilcoxon. * ${ }^{*}$ Prueba exacta de Fisher. 
Tabla 2: Tiempo de tolerancia a la desaturación a 90\% y tiempo en recuperar la saturación a 94\%.

\begin{tabular}{|c|c|c|c|c|}
\hline Variable & $\begin{array}{l}\text { Grupo 1: Triflo } \\
\qquad(N=12)\end{array}$ & $\begin{array}{l}\text { Grupo 2: control } \\
\qquad(\mathrm{N}=12)\end{array}$ & $\begin{array}{l}\text { Diferencia } \\
\left(\mathrm{IC}_{95 \%}\right)\end{array}$ & $p^{*}$ \\
\hline Tiempo a la desaturación a 90\% & $148.83 \pm 14.35$ & $113.08 \pm 20.43$ & $35.75(-50.70--20.80)$ & $<0.0001$ \\
\hline
\end{tabular}

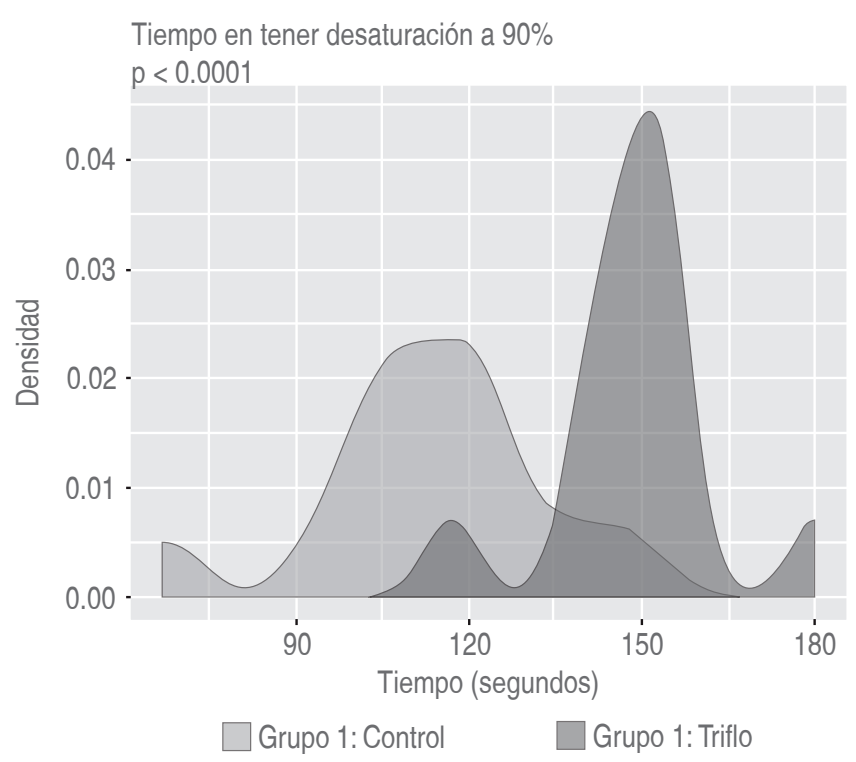

Figura 1: Tiempo en tener desaturación a 90\%.

vamente su FC y PAM. Al conectar el tubo orotraqueal al circuito fueron medidos el $\mathrm{EtCO}_{2}$ inicial, la distensibilidad pulmonar, las resistencias de la vía aérea y el tiempo en recuperar la saturación a $94 \%$. A la hora de realizada la intubación, se recolectaron una vez más los datos de distensibilidad pulmonar y resistencias de la vía aérea. La ventilación mecánica fue en modo control por volumen, con un PEEP igual o mayor a 5 , volumen corriente $6-8 \mathrm{~mL} / \mathrm{kg}$ calculado con el peso predicho, el resto de los parámetros fueron elegidos por adscrito de anestesiología en sala.

Se vaciaron los datos en la hoja de recolección de Excel (versión 2013 para Windows), incluyendo las variables previamente descritas.

Las variables categóricas fueron descritas como frecuencia absoluta y porcentaje. Las variables continuas fueron sometidas a pruebas de normalidad (Shapiro-Wilk). Las variables con distribución normal fueron descritas en términos de media y desviación estándar; las variables con distribución no paramétrica y las variables ordinales fueron descritas

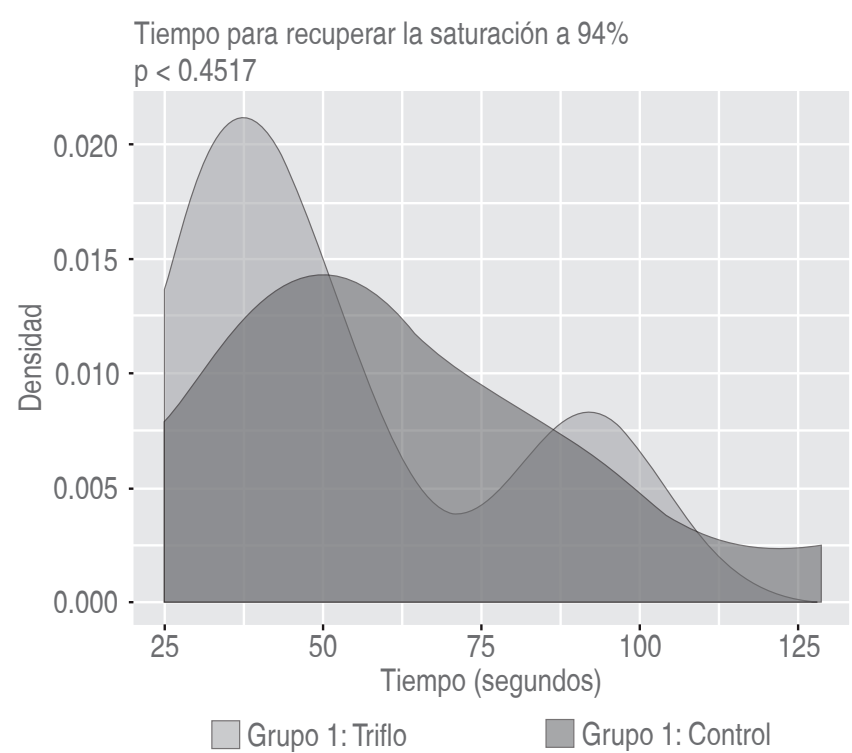

Figura 2: Tiempo para recuperar la saturación a 94\%.

como mediana, rango intercuartil mínimo y máximo. La comparación entre variables continuas con distribución normal se realizó con la prueba t de Student a dos colas; para las variables no paramétricas con una prueba de suma de rangos de Wilcoxon. Un valor de $\mathrm{p}$ a dos colas $\leq 0.05$ se consideró estadísticamente significativo.

Una vez terminada la captura de datos, se importó al programa estadístico R 3.5.0 $0^{13}$ «Joy in Playing» (A Language and Environment for Statistical Computing. $R$ Core Team. $R$ Foundation for Statistical Computing. Vienna, Austria. ISBN 3-900051-07-0 [https://www.R-project.org]) en 4.

\section{RESULTADOS}

Los 24 pacientes en estudio se clasificaron como ASA II, 12 realizaron espirometría incentiva preoperatoria (grupo 1), el resto fue denominado como grupo control. La media de edad para el grupo 1 fue de 56.75 , para el grupo control fue de 40, en ambos gru- 
An Med ABC. 2021; 66 (3): 169-176

pos fueron cinco pacientes mujeres y siete hombres, la media del IMC para el grupo 1 fue de 32.20 y para el grupo control de 32.71. Sólo se registraron pacientes con tabaquismo activo en el grupo control con un total de tres (25\%). $\mathrm{El} \mathrm{EtCO}_{2}$ inicial que se obtuvo al momento de conectar el tubo endotraqueal a la máquina de anestesia registró una media para el grupo 1 de 34.67, la cual es menor que la media del grupo control con 37.25. Otra diferencia fue el PEEP asignado para el grupo 1 con media de 6.33, el grupo control reportó una media de 6.67. El resto de las características de la población se presentan en la Tabla 1.

El tiempo de tolerancia a la desaturación a $90 \%$ fue mayor en los pacientes del grupo 1 presentando una media en segundos de 148.83, comparada con la media del grupo control, la cual fue de 113.08, estos datos muestran una p significativa de $<0.0001$ ( $T a$ bla 2). Para el tiempo en recuperar una saturación a 94\% el grupo 1 obtuvo una mediana de 41, mientras que el grupo control tuvo una mediana de 55 . Al poner en contraste la Figura 1 con la Figura 2 podemos diferenciar de manera más sencilla la distribución de los pacientes del grupo 1, quienes tienden a desaturar en mayor tiempo, mientras que al momento de recuperar la saturación a $94 \%$ la muestra es mucho más heterogénea, presentando dos picos, uno alrededor de los 30 segundos y otro a los 90 .
En las Tablas 3 y 4 se muestran los cambios hemodinámicos y las diferencias entre variables durante el periodo de apnea.

Los cambios en la distensibilidad pulmonar y en las resistencias de la vía aérea, así como las diferencias entre variables se muestran en las Tablas 5 y 6 .

Los datos obtenidos para recuperar una saturación a 94\% muestran en un inicio ser favorables para los pacientes que realizaron espirometría incentiva preoperatoria, ya que la mediana es de 41 , mientras que en el grupo control es de 55; sin embargo, la distribución de los pacientes no es homogénea, tal como se muestra en la Figura 2, teniendo el grupo 1 otro pico de individuos que tardaron hasta 90 segundos en recuperar la saturación a $94 \%$. No hubo diferencias significativas entre el grupo $1 \mathrm{y}$ el grupo control para los cambios hemodinámicos durante la apnea. La distensibilidad pulmonar postintubación fue mayor en el grupo control (grupo 1: media de 50.75; grupo control: media de 56.83), respecto a las resistencias de la vía aérea postintubación, el grupo control también tuvo mejores resultados (grupo 1: mediana de 8.5; grupo control: mediana de 6.5) mencionadas en las Tablas 5 y 6 ; sin embargo, al medir nuevamente la distensibilidad pulmonar (DP) y las resistencias de la vía aérea (RVA) una hora después de la intubación, hubo mayores cambios en el grupo control (gru-

Tabla 3: Cambios hemodinámicos durante el periodo de apnea.

\begin{tabular}{|c|c|c|c|c|}
\hline Variable & Ingreso a quirófano & Apnea & Cambio & $p^{*}$ \\
\hline \multicolumn{5}{|l|}{ Grupo 1} \\
\hline FC & $71.67 \pm 13.06$ & $79.33 \pm 11.27$ & $-7.67(-17.42-2.09)$ & 0.1116 \\
\hline \multicolumn{5}{|l|}{ Grupo 2} \\
\hline FC & $75.83 \pm 17.82$ & $79.75 \pm 15.19$ & $-3.92(-11.28-3.45)$ & 0.2664 \\
\hline TAM & $107.00 \pm 9.14$ & $94.75 \pm 12.90$ & $12.25(2.62-21.88)$ & 0.0173 \\
\hline
\end{tabular}

Tabla 4: Diferencia entre variables hemodinámicas durante el periodo de apnea.

\begin{tabular}{lcccc}
\hline Variable & $\begin{array}{c}\text { Grupo 1: Triflo } \\
(\mathrm{N}=12)\end{array}$ & $\begin{array}{c}\text { Grupo 2: control } \\
(\mathrm{N}=12)\end{array}$ & $\begin{array}{c}\text { Diferencia } \\
\left(\mathrm{IC}_{95 \%}\right)\end{array}$ & $\mathrm{p}^{*}$ \\
\hline FC ingreso a quirófano & $71.67 \pm 13.06$ & $75.83 \pm 17.82$ & $-4.16(-9.06-17.39)$ & 0.5203 \\
TAM ingreso a quirófano & $109.83 \pm 20.81$ & $107.00 \pm 9.14$ & $2.83(-16.81-11.14)$ & 0.6719 \\
FC apnea & $79.33 \pm 11.27$ & $79.75 \pm 15.19$ & $-0.42(-10.91-11.74)$ & 0.9399 \\
TAM apnea & $103 \pm 18.68$ & $94.75 \pm 12.90$ & $8.25(-21.84-5.34)$ & 0.2212 \\
\hline
\end{tabular}

Valores presentados como: media \pm desviación estándar. $\mathrm{FC}=$ frecuencia cardiaca; TAM = tensión arterial media.

*Prueba t de Student. 
Tabla 5: Cambios en distensibilidad pulmonar y resistencias de la vía aérea.

\begin{tabular}{|c|c|c|c|c|}
\hline Variable & Postintubación & Hora de intubación & Cambio & $\mathrm{p}^{*}$ \\
\hline \multicolumn{5}{|l|}{ Grupo 1} \\
\hline DP & $50.75 \pm 9.33$ & $46.42 \pm 9.87$ & $4.33(-1.68-10.35)$ & 0.1413 \\
\hline \multicolumn{5}{|l|}{ Grupo 2} \\
\hline DP & $56.83 \pm 14.44$ & $44.08 \pm 12.47$ & $12.75(5.89-19.61)$ & 0.0018 \\
\hline RVA & $6.5(1.50,5-11)$ & $7(3,6-14)$ & -0.5 & 0.0077 \\
\hline
\end{tabular}

Tabla 6: Diferencia entre variables de distensibilidad pulmonar y resistencias de la vía aérea.

\begin{tabular}{lcccc}
\hline Variable & Grupo 1: Triflo $(\mathrm{N}=12)$ & Grupo 2: control $(\mathrm{N}=12)$ & ${\text { Diferencia }\left(\mathrm{IC}_{95 \%}\right)} \mathrm{p}^{*}$ & 0.2333 \\
DPPI & $50.75 \pm 9.33$ & $56.83 \pm 14.44$ & $-6.08(-4.21-16.38)$ & 0.4801 \\
RVAPI & $8.5(3,5-12)$ & $6.5(1.50,5-11)$ & 2 & 0.6163 \\
DPHI & $46.42 \pm 9.87$ & $44.08 \pm 12.47$ & $2.34(-11.85-7.19)$ & 0.2887 \\
RVAHI & $8.5(2.25,6-11)$ & $7(3,6-14)$ & 1.5 & \\
\hline
\end{tabular}

Valores presentados como: media \pm desviación estándar; mediana (RIC, mín.-máx.). DPPI = distensibilidad pulmonar postintubación; RVAPI = resistencias de la vía aérea postintubación; DPHI = distensibilidad pulmonar a la hora de la intubación, RVAHI = resistencias de la vía aérea a la hora de la intubación.

* Prueba t pareada, rangos señalados de Wilcoxon.

po 1: DP cambio de 4.33, RVA cambio de 0.0; grupo control: DP cambio de 12.75, RVA cambio de 0.5). A pesar de la información obtenida, no se pueden reportar diferencias significativas en ambos grupos.

\section{DISCUSIÓN}

Se han realizado varios estudios para optimizar el estado físico del paciente obeso y lograr un mejor desenlace, ya sea durante la inducción anestésica, transanestésico, extubación o postoperatorio inmediato. Nosotros en esta ocasión decidimos enfocarnos en el apartado de la inducción. Como hemos revisado a lo largo del trabajo, la mecánica ventilatoria en el paciente obeso juega en contra, principalmente viéndose reducida la CFR, desde el punto de vista anestésico, esta limitante se añade a la lista de contras que aumentan los factores de riesgo para alguna complicación durante la inducción, ya que disminuye directamente el tiempo en que nuestro paciente tardará en desaturarse, si a esto le agregamos una vía aérea difícil de ventilar y/o intubar, como ocurre con la mayoría de este tipo de pacientes, el escenario se torna aún más complicado. De acuerdo con Jense HG y su equipo, ${ }^{8}$ los pacientes con obesidad tardan en desaturar a $90 \% 247 \pm 21$ segundos, si lo comparamos con los resultados de nuestro grupo control tenemos una gran diferencia con una media de tan solo 113.08 segundos; sin embargo, en nuestro estudio sólo se preoxigenó al paciente durante tres minutos, mientras que en el trabajo citado fue durante cinco minutos. Lo importante y que cabe destacar de nuestros resultados es la p significativa $(<0.0001)$ para la desaturación a $90 \%$ entre el grupo 1 (media 148.83) y el grupo control (media 113.08), mostrando una diferencia de 35.75 segundos con un intervalo de confianza del $95 \%$. Con esto, la hipótesis se comprueba y se cumple el objetivo principal.

Podemos considerar algunos sesgos en el estudio. Empleamos varias estrategias de preoxigenación descritas para el paciente obeso que pudieron manejarse por separado. Rovira y sus colegas ${ }^{15}$ nos recomiendan una preoxigenación con una $\mathrm{FiO}_{2}$ del 0.8 , ya que consigue los mismos efectos beneficiosos y reduce los efectos deletéreos sobre la formación de atelectasias que provoca la preoxigenación con una $\mathrm{FiO}_{2}$ de 1.0. También efectuamos el uso de CPAP con $10 \mathrm{cmH}_{2} \mathrm{O}$; sin embargo, Nimmagadda $\mathrm{U}$ y su grupo $^{16}$ mencionan que el uso de CPAP por sí solo durante la preoxigenación de pacientes obesos no retrasa el inicio de la desaturación, porque la capacidad funcional residual volvió al nivel pre-CPAP cuando el paciente estaba inducido y la máscara fue eliminada, la diferencia fue que nosotros mantuvimos la mascarilla colocada hasta que la intubación 
fuese realizada. Todos los pacientes fueron colocados en rampa o posición de HELP, la cual ha demostrado que prolonga el tiempo de desaturación en aproximadamente 50 segundos de acuerdo con lo publicado con Dixon BJ y colaboradores. ${ }^{14}$ Todo se hizo con el fin de brindar la mejor atención al paciente tal como lo indica el centro médico donde se desarrolló el trabajo. La preoxigenación fue la misma en el grupo 1 y en el grupo control.

Se espera que el trabajo tenga repercusiones positivas en otras poblaciones, con futuros estudios en pacientes obesos mórbidos e incluso en personas con IMC normal, pudiendo incorporar a las indicaciones el uso de Triflo preoperatorio de manera rutinaria a todos los pacientes sometidos a anestesia general con intubación orotraqueal.

\section{CONCLUSIONES}

La obesidad es un problema grave de salud en nuestro país, no distingue entre estrato social, tipo de ascendencia, edad o sexo. Como anestesiólogos, y antes que nada como médicos, buscamos siempre el bienestar de nuestro paciente; por lo tanto, este trabajo es para brindar una herramienta extra y útil a los encargados del cuidado de la salud, no sólo al Departamento de Anestesiología, sino a todos aquellos que interactúan con el paciente obeso quirúrgico en su valoración preanestésica: cirujanos generales, cirujanos bariátricos, internistas, intensivistas, cardiólogos, endocrinólogos, etcétera.

Se ha demostrado que el uso de espirometría incentiva preoperatoria a través del empleo del dispositivo Triflo con una hora mínimo antes de la inducción de la anestesia, es eficaz para mejorar el tiempo de tolerancia a la desaturación a $90 \%$ en pacientes con obesidad sometidos a cirugía electiva en el Centro Médico ABC.

Los pacientes que realizan espirometría incentiva preoperatoria no recuperan más rápido una saturación a $94 \%$ en comparación con los pacientes que no la efectúan.

El uso de Triflo preoperatorio no modifica los cambios hemodinámicos (FC y PAM) durante la apnea en comparación con los pacientes que no utilizan Triflo preoperatorio.

El uso de Triflo preoperatorio no modifica la distensibilidad pulmonar ni las resistencias de la vía aérea postintubación en pacientes programados para cirugía electiva en comparación con los pacientes que no utilizan Triflo preoperatorio. Sin embargo, una hora después de la intubación, hay menos cambios en los pacientes que lo utilizaron.

\section{REFERENCIAS}

1. NORMA Oficial Mexicana NOM-008-SSA3-2010, Para el tratamiento integral del sobrepeso y la obesidad [Internet]. Gob.mx. [citado el 18 de marzo de 2018]. Disponible en: http:// www.dof.gob.mx/normasOficiales/4127/Salud/Salud.htm

2. Auler JO Jr, Miyoshi E, Fernandes CR, Benseñor FE, Elias L, Bonassa J. The effects of abdominal opening on respiratory mechanics during general anesthesia in normal and morbidly obese patients: a comparative study. Anesth Analg. 2002; 94 (3): 741-748.

3. Vieito Amor M, Hernández Iniesta J, Santiveri X, García CH, Maestre P, Villalonga A et al. Morbimortalidad anestésicaquirúrgica en 60 pacientes intervenidos de cirugía bariátrica. Rev Esp Anestesiol Reanim. 2002; 49: 365-372.

4. Montoya PT, Borunda ND, Domínguez CG. Manejo anestésico en el paciente obeso mórbido sometido a cirugía bariátrica. Rev Mex Cir Endoscop. 2008; 9 (4): 188-193.

5. Dumont L, Mattys M, Mardirosoff C, Vervloesem N, Allé JL, Massaut J. Changes in pulmonary mechanics during laparoscopic gastroplasty in morbidly obese patients. Acta Anaesthesiol Scand. 1997; 41 (3): 408-413.

6. Pelosi P, Croci M, Ravagnan I, Tredici S, Pedoto A, Lissoni A et al. The effects of body mass on lung volumes, respiratory mechanics, and gas exchange during general anesthesia. Anesth Analg. 1998; 87 (3): 654-660.

7. Rothen HU, Neumann P, Berglund JE, Valtysson J, Magnusson A, Hedenstierna G. Dynamics of re-expansion of atelectasis during general anaesthesia. Br J Anaesth. 1999; 82 (4): 551-556.

8. Jense HG, Dubin SA, Silverstein PI, O'Leary-Escolas U. Effect of obesity on safe duration of apnea in anesthetized humans. Anesth Analg. 1991; 72 (1): 89-93.

9. Soro Domingo M, Belda Nácher FJ, Aguilar Aguilar G, Ferrandis Comes R, García-Raimundo M, Martínez Ponsa V. Preoxigenación en anestesia. Rev Esp Anestesiol Reanim. 2004; 51: 322-327.

10. Levitan R, Ochroch EA. Airway management and direct laryngoscopy. a review and update. Crit Care Clin. 2000; 16 (3): 373-388.

11. Carrillo-Esper R, de los Monteros-Estrada IE, RosalesGutiérrez O, García-Martínez AJ, Baeza-García AD, CarrilloCórdova DM et al. Posición en rampa. Rev Mex Anest. 2013; 36 (3): 209-211.

12. Restrepo RD, Wettstein R, Wittnebel L, Tracy M. Incentive spirometry: 2011. Respir Care. 2011; 56 (10): 1600-1604.

13. Agostini P, Singh S. Incentive spirometry following thoracic surgery: what should we be doing? Physiotherapy. 2009; 95 (2): $76-82$.

14. Dixon BJ, Dixon JB, Carden JR, Burn AJ, Schachter LM, Playfair JM et al. Preoxygenation is more effective in the 25 degrees head-up position than in the supine position in severely obese patients: a randomized controlled study. Anesthesiology. 2005; 102 (6): 1110-1115; discussion 5A.

15. Rovira L. Efectos de un programa de fisioterapia respiratoria preoperatoria sobre la oxigenación y la función respiratoria en pacientes obesos mórbidos sometidos a cirugía bariátrica laparoscópica [Tesis Doctoral]. España: Universidad de Valencia. Facultad de Medicina y Odontología; 2014.

16. Nimmagadda U, Salem MR, Crystal GJ. Preoxygenation: physiologic basis, benefits, and potential risks. Anesth Analg. 2017; 124 (2): 507-517. 